\title{
WEIGHTED INEQUALITIES FOR CONVOLUTIONS
}

\author{
KENNETH F. ANDERSEN
}

(Communicated by Andrew Bruckner)

\begin{abstract}
For certain convolution operators $T$ on $R^{+}$or $R^{n}$, sufficient conditions are given which ensure that $T$ is bounded between weighted Lebesgue spaces. The class of operators considered includes many of classical interest; in particular, new inequalities are obtained for the Laplace transform, the Poisson integral on $R^{n} \times R^{+}$, and Goldberg's transform.
\end{abstract}

\section{INTRODUCTION}

In a recent paper Bloom [2] obtained weighted inequalities for the Laplace transform $\mathscr{L}$ using known weighted inequalities for the Hardy operator. The purpose of this paper is twofold. First, we show that these weighted Hardy inequalities may be used in a different and, we think, more elementary way to obtain weighted inequalities for a class of operators on $R^{+}=(0, \infty)$. Our results will be seen to include and sharpen Bloom's results for $\mathscr{L}$. Secondly, we show that our approach admits a natural extension to a class of operators on $R^{n}$; applications will be illustrated by deriving new inequalities for Goldberg's transform on $R$ and the Poisson integral on the half space $R^{n+}=R^{n} \times R^{+}$.

If $X$ denotes $R^{+}$or $R^{n}$ and $\mu$ is a positive Borel measure on $X$, denote by $L^{p}(X, \mu)$ the weighted Lebesgue space of measurable functions $f$ on $X$ for which $\|f\|_{p, \mu}<\infty$ where

$$
\|f\|_{p, \mu}= \begin{cases}\left(\int_{X}|f|^{p} d \mu\right)^{1 / p} & \text { if } 1 \leq p<\infty, \\ \mu \text { ess. } \sup _{y \in X}|f(y)| & \text { if } p=\infty .\end{cases}
$$

If $f(y)$ is expressed as a formula in $y$ it will sometimes be convenient to slightly abuse the notation $\|f\|_{p, \mu}$ by writing instead $\|f(y)\|_{p, \mu}$.

The operators considered here are convolutions with a suitable kernel $k$ and are given by

$$
T f(x)= \begin{cases}\int_{R^{+}} k(x / y) f(y) d y / y & \text { if } X=R^{+}, \\ \int_{R^{n}} k(|x-y|) f(y) d y & \text { if } X=R^{n} .\end{cases}
$$

Received by the editors July 1, 1993.

1991 Mathematics Subject Classification. Primary 26D15.

Key words and phrases. Weighted inequalities, convolution, integral operator.

Research supported in part by NSERC grant \#OGP8185. 
Given such $T$ and exponents $1 \leq p, q \leq \infty$, sufficient conditions to be satisfied by positive Borel measures $\mu$ and $\nu$ are given which ensure that

$$
\|T f\|_{q, \mu} \leq C\|f\|_{p, \nu}
$$

for a constant $C$ independent of $f \in L^{p}(X, \nu)$. Since $\nu=\nu_{a}+\nu_{s}$ where $\nu_{a}$ is absolutely continuous and $\nu_{s}$ is singular with respect to Lebesgue measure on $X$, there is no loss of generality in taking $\nu=\nu_{a}$ since the left side of (1.2) is unchanged if $f$ is redefined to be zero on the support of $\nu_{s}$. For convenience we set $v=d \nu_{a} / d x$ and $d \sigma / d x=v^{-1 /(p-1)}$. As usual $p^{\prime}$ denotes the conjugate exponent of $p$ given by $1 / p+1 / p^{\prime}=1$ and if $q<p$ the exponent $r$ is defined by $1 / r=1 / q-1 / p$. We define a function $\omega(t)=\omega(X ; \mu, \nu ; q, p ; t)$ for $t>0$ as follows, $\chi_{E}$ denoting the characteristic function of $E$.

If $X=R^{+}$,

$$
\omega(t)= \begin{cases}\sup _{s>0}\left\|\chi_{(0, s t]}\right\|_{q, \mu}\left\|y^{-1} \chi_{[s, \infty)}(y)\right\|_{p^{\prime}, \sigma} & \text { if } p \leq q, \\ \left(\int_{0}^{\infty}\left[\left\|\chi_{(0, s t]}\right\|_{q, \mu}\left\|y^{-1} \chi_{[s, \infty)}(y)\right\|_{p^{\prime}, \sigma}^{p^{\prime} / q^{\prime}}\right]^{r} s^{-p^{\prime}} d \sigma(s)\right)^{1 / r} & \text { if } q<p .\end{cases}
$$

If $X=R^{n}, Q$ denotes a cube in $R^{n}$ with centre $x_{Q}$ and sides parallel to the coordinate axis of length $\ell(Q)$. If $j=\left(j_{1}, \ldots, j_{n}\right)$ for integers $j_{1}, \ldots, j_{n}$, let $Q_{j}$ denote the translate of $Q$ by $\ell(Q) j$ so that $x_{Q_{j}}=x_{Q}+\ell(Q) j$ and $\ell\left(Q_{j}\right)=\ell(Q)$. Let $Q^{*}$ denote the dilate of $Q$ by the factor 3 so that $x_{Q^{*}}=x_{Q}$ and $\ell\left(Q^{*}\right)=3 \ell(Q)$. Set $Q_{j}^{*}=\left(Q_{j}\right)^{*}$. Then

$$
\omega(t)=\inf _{\{Q: \ell(Q)=t\}} \omega_{Q}
$$

where

$$
\omega_{Q}= \begin{cases}\sup _{j}\left\|\chi_{Q_{j}}\right\|_{q, \mu}\left\|\chi_{Q_{j}^{*}}\right\|_{p^{\prime}, \sigma} & \text { if } p \leq q, \\ \left(\sum_{j}\left[\left\|\chi_{Q_{j}}\right\|_{q, \mu}\left\|\chi_{Q_{j}^{*}}\right\|_{p^{\prime}, \sigma}\right]^{r}\right)^{1 / r} & \text { if } q<p .\end{cases}
$$

Throughout, we adopt the usual convention that products of the form $0 \cdot \infty$ are taken to be zero and when $p=1$ or $p=\infty$, expressions involving $\sigma$ are interpreted as appropriate limits; for example, $\left\|y^{-1} \chi_{[s, \infty)}(y)\right\|_{p^{\prime}, \sigma}$ is taken to be ess. $\sup _{y \in[s, \infty)}[y v(y)]^{-1}$ when $p=1$. Note that $w(t)$ is nonnegative, nondecreasing on $R^{+}$and is continuous wherever it is finite. In particular, $\omega(\infty)=\lim _{t \rightarrow \infty} \omega(t)$ exists as an extended real number.

If $k(t)$ is a nonnegative, nonincreasing, right continuous function on $R^{+}$, set $k(\infty)=\lim _{t \rightarrow \infty} k(t)$ and denote by $\Lambda_{k}$ the positive Borel measure on $R^{+}$ generated by $k$ defined by $\Lambda_{k}(a, b]=k(a)-k(b)$; see [10, Chapter 11].

If $k(t)=\chi_{(0,1)}(t)$ the operator given by (1.1) with $X=R^{+}$is the 'dual' Hardy operator $A_{1} f(x)=\int_{x}^{\infty} f(y) d y / y$ for which $\omega(1)<\infty$ is a known (see [8], [1], [3], [7]) necessary and sufficient condition for (1.2) to hold. Moreover, in this case the smallest constant $C$ in (1.2) satisfies $c_{1, p, q} \omega(1) \leq C \leq$ $c_{2, p, q} \omega(1)$ for constants $c_{1, p, q}$ and $c_{2, p, q}$. Our main result generalizes this as follows.

Theorem 1. Let $k(t)$ be nonnegative, nonincreasing, and right continuous on $R^{+}$. Suppose $1 \leq p, q \leq \infty$ and that $\mu, \nu$ are positive Borel measures on $X$ 
with $\omega(t)=\omega(X ; \mu, \nu ; q, p ; t)$ satisfying

$$
K=\int_{R^{+}} \omega(t) d \Lambda_{k}(t)+k(\infty) \omega(\infty)<\infty
$$

Then there is a constant $c_{X, p, q}$ such that (1.2) holds with $C=c_{X, p, q} K$ for all $f \in L^{p}(X, \nu)$.

It should be noted that the integral in (1.3) is equivalent to the improper Riemann-Stieltjes integral $\int_{0}^{\infty} \omega(t) d[-k(t)]$ whenever $k$ and $\omega$ have no common point of discontinuity; in particular, this is the case if $k$ is continuous or if $\omega(t)<\infty$ for all $t$.

With $X=R^{+}$and each of $\mu$ and $\nu$ of the form $d \mu / d x=x^{\alpha}$, inequalities of the form (1.2) for various choices of $k$ have been given in Chapter 9 of [4], see especially Theorems 319, 341(2), 342(2), and 360. Although Theorem 1 generalizes many of these results, here we elaborate only the case $k(t)=e^{-t}$ which leads by a change of variable to new inequalities for the Laplace transform $\mathscr{L}$ since in that case $\mathscr{L} f(x)=T g(x)$ where $g(y)=y^{-1} f\left(y^{-1}\right)$.

Corollary 1. If $1 \leq p, q \leq \infty$, and $\mu, \nu$ are positive Borel measures on $R^{+}$ with $K<\infty$ where

$$
K= \begin{cases}\int_{0}^{\infty} e^{-t} \sup _{s>0}\left\|\chi_{(0, t / s]}\right\|_{q, \mu}\left\|\chi_{(0, s]}\right\|_{p^{\prime}, \sigma} d t & \text { if } p \leq q, \\ \int_{0}^{\infty} e^{-t}\left(\int_{0}^{\infty}\left[\left\|\chi_{(0, t / s]}\right\|_{q, \mu}\left\|\chi_{(0, s]}\right\|_{p^{\prime}, \sigma}^{p^{\prime} / q^{\prime}}\right]^{r} d \sigma(s)\right)^{1 / r} d t & \text { if } q<p\end{cases}
$$

then there is a constant $c_{p, q}$ such that

$$
\|\mathscr{L} f\|_{q, \mu} \leq C\|f\|_{p, \nu}
$$

with $C=c_{p, q} K$ for all $f \in L^{p}\left(R^{+}, \nu\right)$.

Bloom [2] proved two theorems, each giving a sufficient condition and a different necessary condition for (1.5) to hold with $d \mu=u d x, d \nu=v d x$, and $1<p, q<\infty$. In particular, his second theorem asserts that if $B_{\delta}$ is defined by

$$
B_{\delta}= \begin{cases}\sup _{s>0}(\mathscr{L} u(\delta s))^{1 / q}\left\|\chi_{(0, s]}\right\|_{p^{\prime}, \sigma} & \text { if } p \leq q, \\ \left(\int_{0}^{\infty}\left[(\mathscr{L} u(\delta s))^{1 / q}\left\|\chi_{(0, s]}\right\|_{p^{\prime}, \sigma}^{p^{\prime} / q^{\prime}}\right]^{r} d \sigma(s)\right)^{1 / r} & \text { if } q<p,\end{cases}
$$

then $B_{1}<\infty$ is sufficient while $B_{q}<\infty$ is necessary for (1.5) to hold. Since

$$
\int_{0}^{t / s} u \leq e^{\delta t} \int_{0}^{t / s} e^{-\delta s x} u(x) d x \leq e^{\delta t} \mathscr{L} u(\delta s)
$$

it follows immediately that $K<\infty$ in (1.4) if $B_{\delta}<\infty$ for some $\delta<q$. Thus Corollary 1 contains the sufficient condition and narrows the gap between the necessary and the sufficient conditions of Bloom's Theorem 2. Similarly, it is not difficult to show that Corollary 1 also contains the sufficient condition of his Theorem 1.

Among other applications, Theorem 1 may be used to obtain inequalities for the operators given by the Gauss-Weierstrass kernel $W_{y}(x)=(4 \pi y)^{-n / 2} e^{-|x| / 4 y}$ and the Poisson kernel $P_{y}(x)=\Gamma\left(\frac{n+1}{2}\right) y\left[\pi\left(y^{2}+|x|^{2}\right)\right]^{-(n+1) / 2}$ where $(x, y) \epsilon$ $R^{n+}$, for which inequalities of the form (1.2) have been widely studied. In particular, we have the following result for the Poisson operator. 
Corollary 2. If $1 \leq p, q \leq \infty, y>0$, and $\mu, \nu$ are positive Borel measures on $R^{n}$ with $\omega(t)=\omega\left(R^{n} ; \mu, \nu ; q, p ; t\right)$ satisfying

$$
\int_{1}^{\infty} \frac{\omega(t)}{t^{n+2}} d t<\infty
$$

then

$$
\left\|\int_{R^{n}} P_{y}(x-z) f(z) d z\right\|_{q, \mu} \leq C_{y}\|f\|_{p, \nu}
$$

with

$$
C_{y}=\frac{3^{n / p}(n+1) \Gamma\left(\frac{n+1}{2}\right)}{\pi^{(n+1) / 2}} y \int_{0}^{\infty} \frac{t \omega(t)}{\left(y^{2}+t^{2}\right)^{(n+3) / 2}} d t .
$$

A simple calculation shows that for $1<p=q<\infty$ and $d \mu / d x=d \nu / d x=$ $(1+|x|)^{\alpha}$,

$$
\omega(t) \leq c_{n, p, \alpha} \begin{cases}t^{n}(1+t)^{\max \left(0,-n / p^{\prime}+\alpha / p\right)}, & \alpha \geq 0, \\ t^{n}(1+t)^{\max (0,-n / p-\alpha / p)}, & \alpha<0,\end{cases}
$$

and hence (1.7) holds in this case if $-n-p<\alpha<n(p-1)+p$. Moreover, this range of $\alpha$ is best possible for (1.7); the necessity of $-n-p<\alpha$ follows by taking $f(z)=|z| / \log |z|$ for large $|z|$ and $f(z)=0$ otherwise. A duality argument shows the necessity of $\alpha<n(p-1)+p$.

Note that if $1 \leq p \leq q \leq \infty$ and there is a constant $K$ with

$$
\left\|\chi_{Q}\right\|_{q, \mu}\left\|\chi_{Q}\right\|_{p^{\prime}, \sigma} \leq K[\ell(Q)]^{n}
$$

for all cubes $Q$, then $\omega(t) \leq K(3 t)^{n}$ so Corollary 2 shows that (1.7) holds with constant $C$ independent of $y$; the case $n=1 \leq p=q<\infty$ was obtained by Muckenhoupt [9, Theorem 2].

The condition (1.9) with $n=1<p=q<\infty$ and $d \mu / d x=d \nu / d x=v$ is the well-known $A_{p}$ condition which characterizes [6] the weight functions $v$ for which the Hilbert transform

$$
H f(x)=\text { p.v. } \frac{1}{\pi} \int_{R} \frac{f(t)}{x-t} d t, \quad x \in R,
$$

satisfies

$$
\int_{R}|H f|^{p} v \leq C \int_{R}|f|^{p} v
$$

for a constant $C$ independent of $f$. Combining this with Corollary 2 we prove, in section 3, the following weighted inequalities for the Goldberg transform [5] given by

$$
G f(x)=\text { p.v. } \frac{1}{\pi} \int_{R} \frac{\sin (x-t)}{(x-t)^{2}} f(t) d t, \quad x \in R .
$$

Theorem 2. If $1<p<\infty$ and $v=d \nu / d x$ is a weight function on $R$ with $\omega(t)=\omega(R ; \nu, \nu ; p, p ; t)$ satisfying

$$
\frac{\omega(t)}{t}+\int_{1}^{\infty} \frac{\omega(s)}{s^{3}} d s \leq K
$$


for some constant $K$ and all $0<t<1$, then there is a constant $C$ depending on $p$ and $K$ such that

$$
\int_{R}|G f|^{p} v \leq C \int_{R}|f|^{p} v
$$

for all $f \in L^{p}(R, \nu)$.

Theorem 1 is proved in section 2 . Constants are denoted by $c$ or $C$, with or without subscripts, but are not necessarily the same from line to line.

\section{Proof of Theorem 1}

We prove the case $X=R^{+}$first.

For $t>0$ let the Hardy operators $A_{t}$ be given by

$$
A_{t} f(x)=\int_{x / t}^{\infty} f(y) \frac{d y}{y}, \quad x \in R^{+} .
$$

As noted in the introduction, $A_{1}$ satisfies

$$
\left\|A_{1} f\right\|_{q, \mu} \leq C\|f\|_{p, \nu}
$$

with $C=c_{2, p, q} \omega\left(R^{+} ; \mu, \nu ; q, p ; 1\right)$ and hence a change of variable shows

$$
\left\|A_{t} f\right\|_{q, \mu} \leq c_{2, p, q} \omega\left(R^{+} ; \mu, \nu ; q, p ; t\right)\|f\|_{p, \nu} \text {. }
$$

Suppose now that $f \geq 0$ and let $T_{1}$ be the operator associated with the kernel $k_{1}(t)=k(t)-k(\infty)$. Then $k_{1}(\infty)=0$ and hence Fubini's Theorem shows

$$
\begin{aligned}
T_{1} f(x) & =\int_{0}^{\infty} f(y) \int_{(x / y, \infty)} d \Lambda_{k}(t) \frac{d y}{y} \\
& =\int_{R^{+}} d \Lambda_{k}(t) \int_{x / t}^{\infty} f(y) \frac{d y}{y} \\
& =\int_{R^{+}} A_{t} f(x) d \Lambda_{k}(t) .
\end{aligned}
$$

Minkowski's inequality for integrals now yields

$$
\begin{aligned}
\left\|T_{1} f\right\|_{q, \mu} & \leq \int_{R^{+}}\left\|A_{t} f\right\|_{q, \mu} d \Lambda_{k}(t) \\
& \leq c_{2, p, q}\left(\int_{R^{+}} \omega(t) d \Lambda_{k}(t)\right)\|f\|_{p, \nu}
\end{aligned}
$$

in view of (2.1).

On the other hand, Hölder's inequality shows

$$
\begin{aligned}
\left\|\left(T-T_{1}\right) f\right\|_{q, \mu} & =k(\infty)\left\|\chi_{R^{+}}\right\|_{q, \mu} \int_{R^{+}}|f(y)| \frac{d y}{y} \\
& \leq k(\infty)\left\|\chi_{R^{+}}\right\|_{q, \mu}\left\|y^{-1} \chi_{R^{+}}(y)\right\|_{p^{\prime}, \sigma}\|f\|_{p, \nu} \\
& =k(\infty) c_{p, q} \omega(\infty)\|f\|_{p, \nu}
\end{aligned}
$$

where $c_{p, q}=1$ if $p \leq q$ and $c_{p, q}=\left(r / p^{\prime}\right)^{1 / r}$ otherwise. This combined with (2.2) completes the proof for the case $X=R^{+}$. 
Turning to the case $X=R^{n}$, we first prove that for $t>0$ the operator

$$
A_{t} f(x)=\int_{|x-y|<t} f(y) d y
$$

satisfies

$$
\left\|A_{t} f\right\|_{q, \mu} \leq 3^{n / p} C\|f\|_{p, \nu}
$$

with $C=\omega\left(R^{n} ; \mu, \nu ; q, p ; t\right)$.

To prove (2.3), fix a cube $Q$ with $\ell(Q)=t$. Then for fixed $j$ and $x \in Q_{j}$, we have $\{y:|x-y|<t\} \subset Q_{j}^{*}$ and therefore

$$
\begin{aligned}
\left|A_{t} f(x)\right| & \leq \int_{Q_{j}^{*}}|f(y)| d y \\
& \leq\left\|\chi_{Q_{j}^{*}}\right\|_{p^{\prime}, \sigma}\left\|\chi_{Q_{j}^{*}} f\right\|_{p, \nu}
\end{aligned}
$$

by Hölder's inequality. The case $q=\infty$ of (2.3) follows easily from (2.4) so we give the details only for $q<\infty$. Now, if $q<\infty$, then (2.4) shows

$$
\begin{aligned}
\left\|A_{t} f\right\|_{q, \mu} & \leq\left(\sum_{j} \int_{Q_{j}}\left|A_{t} f\right|^{q} d \mu\right)^{1 / q} \\
& \leq\left(\sum_{j}\left\|\chi_{Q_{j}}\right\|_{q, \mu}^{q}\left\|\chi_{Q_{j}}\right\|_{p^{\prime}, \sigma}^{q}\left\|\chi_{Q_{j}^{*}} f\right\|_{p, \nu}^{q}\right)^{1 / q} \\
& \leq \begin{cases}\left(\sup _{j}\left\|\chi_{Q_{j}}\right\|_{q, \mu}\left\|\chi_{Q_{j}^{*}}\right\|_{p^{\prime}, \sigma}\right)\left(\sum_{j}\left\|\chi_{Q_{j}^{*}} f\right\|_{p, \nu}^{q}\right)^{1 / q} & \text { if } p \leq q, \\
\left(\sum_{j}\left[\left\|\chi_{Q_{j}}\right\|_{q, \mu}\left\|\chi_{Q_{j}^{*}}\right\|_{p^{\prime}, \sigma}\right]^{r}\right)^{1 / r}\left(\sum_{j}\left\|\chi_{Q_{j}^{*}} f\right\|_{p, \nu}^{p}\right)^{1 / p} & \text { if } q<p<\infty, \\
\left(\sum_{j}\left[\left\|\chi_{Q_{j}}\right\|_{q, \mu}\left\|\chi_{Q_{j}^{*}}\right\|_{p^{\prime}, \sigma}\right]^{r}\right)^{1 / r}\left(\sup _{j}\left\|\chi_{Q_{j}^{*}} f\right\|_{p, \nu}\right) & \text { if } q<p=\infty\end{cases}
\end{aligned}
$$

where we used Hölder's inequality with exponents $p / q,(p / q)^{\prime}=r / q$ on the sum in the cases $q<p$. We have $\left(\sum_{j}\left\|\chi_{Q_{j}^{*}} f\right\|_{p, \nu}^{q}\right)^{1 / q} \leq\left(\sum_{j}\left\|\chi_{Q_{j}^{*}} f\right\|_{p, \nu}^{p}\right)^{1 / p}$ for $p \leq q$, and thus, in any case, we obtain

$$
\begin{aligned}
\left\|A_{t} f\right\|_{q, \mu} & \leq \omega_{Q} \begin{cases}\left(\sum_{j}\left\|\chi_{Q_{j}^{*}} f\right\|_{p, \nu}^{p}\right)^{1 / p} & \text { if } p<\infty, \\
\sup _{j}\left\|\chi_{Q_{j}^{*}} f\right\|_{p, \nu} & \text { if } p=\infty\end{cases} \\
& =\omega_{Q} \begin{cases}\left(\int_{R^{n}} \sum_{j} \chi_{Q_{j}^{*}}|f|^{p} d \nu\right)^{1 / p} & \text { if } p<\infty, \\
\|f\|_{p, \nu} & \text { if } p=\infty .\end{cases}
\end{aligned}
$$

Since $\sum_{j} \chi_{Q_{j}^{*}}(y) \leq 3^{n}$ a.e., we obtain (2.3) upon taking the infimum over cubes $Q$ with $\ell(Q)=t$.

Now suppose $f \geq 0$ and let $T_{1}$ be the operator associated with the kernel $k_{1}(t)=k(t)-k(\infty)$. Then Fubini's Theorem yields

$$
\begin{aligned}
T_{1} f(x) & =\int_{R^{n}} f(y) \int_{(|x-y|, \infty)} d \Lambda_{k}(t) d y \\
& =\int_{R^{+}} A_{t} f(x) d \Lambda_{k}(t)
\end{aligned}
$$


and the remainder of the proof is analogous to that of the case $X=R^{+}$using (2.3) in place of $(2.1) ; c_{R^{n}, p, q}=3^{n / p}$ will suffice. The details are omitted.

\section{Proof of Theorem 2}

For each integer $j$, let $\chi_{j}$ and $\chi_{j}^{*}$ denote the characteristic functions of $I_{j}=(j-1, j]$ and $I_{j}^{*}=(j-2, j+1]$ respectively. Then since

$$
\left|\frac{\sin t}{t^{2}}-\frac{1}{t}\right| \leq c, \quad|t| \leq 2,
$$

it follows that

$$
\left|G f(x)-\sum_{j}\left[H\left(f \chi_{j}^{*}\right)(x)\right] \chi_{j}(x)\right| \leq c T|f|(x)
$$

where

$$
T f(x)=\frac{1}{\pi} \int_{R} \frac{f(t)}{1+|x-t|^{2}} d t
$$

Now, let $v_{j}(x)$ have period 6 and be given by

$$
v_{j}(x)=\left\{\begin{array}{ll}
v(x) & \text { if } x \in I_{j}^{*}, \\
v(2 j+2-x) & \text { if } x \in I_{j+3}^{*}
\end{array} .\right.
$$

Since (1.11) implies $\omega(t) / t \leq 4^{3} K$ for $t \leq 3$, by considering separately those intervals $Q$ with $\ell(Q) \leq 3$ and those with $\ell(Q)>3$ it follows that there is a constant $c$ independent of $j$ such that (1.9) holds with $n=1, p=q$, $d \mu / d x=d \nu / d x=v_{j}$, and $K$ replaced by $c K$. Hence, (1.10) shows there is a constant $C$ depending only on $p$ and $K$ such that

$$
\begin{aligned}
\int_{R}\left|\sum_{j}\left[H\left(f \chi_{j}^{*}\right)(x)\right] \chi_{j}(x)\right|^{p} v(x) d x & =\sum_{j} \int_{I_{j}}\left|H\left(f \chi_{j}^{*}\right)(x)\right|^{p} v_{j}(x) d x \\
& \leq \sum_{j} \int_{R}\left|H\left(f \chi_{j}^{*}\right)(x)\right|^{p} v_{j}(x) d x \\
& \leq C \sum_{j} \int_{I_{j}^{*}}|f(x)|^{p} v_{j}(x) d x \\
& \leq 3 C \int_{R}|f(x)|^{p} v(x) d x .
\end{aligned}
$$

On the other hand, Corollary 2 yields

$$
\int_{R}[T|f|(x)]^{p} v(x) d x \leq C \int_{R}|f(x)|^{p} v(x) d x
$$

in view of (1.11). 2.

Combining (3.1) and (3.2) yields (1.12) and completes the proof of Theorem

\section{REFERENCES}

1. K. F. Andersen and B. Muckenhoupt, Weighted weak type Hardy inequalities with applications to Hilbert transforms and maximal functions, Studia Math. 72 (1982), 9-26.

2. S. Bloom, Hardy integral estimates for the Laplace transform, Proc. Amer. Math. Soc. 116 (1992), 417-426. 
3. J. S. Bradley, Hardy inequalities with mixed norms, Canad. Math. Bull. 21 (1978), 405-408.

4. G. H. Hardy, J. E. Littlewood, and G. Pólya, Inequalities, Second Ed., Cambridge Univ. Press, Cambridge, 1967.

5. R. R. Goldberg, An integral transform related to the Hilbert transform, J. London Math. Soc. 35 (1960), 200-204.

6. R. A. Hunt, B. Muckenhoupt, and R. Wheeden, Weighted norm inequalities for the conjugate function and Hilbert transform, Trans. Amer. Math. Soc. 176 (1973), 227-251.

7. V. G. Maz'ja, Sobolev spaces, Springer-Verlag, Heidelberg, 1985.

8. B. Muckenhoupt, Hardy's inequality with weights, Studia Math. 44 (1972), 31-38.

9. Soc. 210 (1975), 225-231.

10. R. Wheeden and A. Zygmund, Measure and integral, Marcel Dekker, New York, 1980.

Department of Mathematical Sciences, University of Alberta, Edmonton, Alberta, CANADA T6G 2G1

E-mail address: kanderse@vega.math.ualberta.ca 